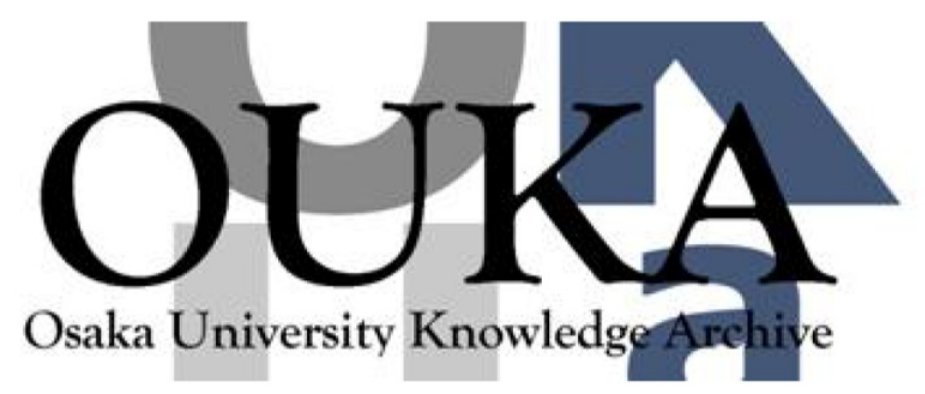

\begin{tabular}{|c|l|}
\hline Title & $\begin{array}{l}\text { Single-shot two-dimensional spectral } \\
\text { interferometry for ultrafast laser-produced } \\
\text { plasmas }\end{array}$ \\
\hline Author(s) & Hama, Y.; Kondo, K.; Zoubir, A. et al. \\
\hline Citation & Optics Letters. 31(12) p. 1917-p. 1919 \\
\hline Issue Date & $2006-06-15$ \\
\hline oaire:version & VoR \\
\hline URL & https://hdl. handle. net/11094/2992 \\
\hline rights & \\
\hline Note & \\
\hline
\end{tabular}

Osaka University Knowledge Archive : OUKA

https://ir. Library. osaka-u. ac. jp/

Osaka University 


\title{
Single-shot two-dimensional spectral interferometry for ultrafast laser-produced plasmas
}

\author{
Y. Hama, K. Kondo, A. Zoubir, T. Honda, R. Kodama, K. A. Tanaka, and K. Mima \\ Graduate School of Engineering, Osaka University, 2-1 Yamadaoka, Suita, 565-0871, Japan, and \\ Institute of Laser Engineering, Osaka University, 2-6 Yamadaoka, Suita, 565-0871, Japan
}

\begin{abstract}
Received February 21, 2006; revised March 31, 2006; accepted April 3, 2006; posted April 10, 2006 (Doc. ID 68049) Coherent white light was used as a light source for spectral interferometry of ultrafast laser-produced plasmas. Using a narrowband filter, two-dimensional images of field ionization in helium were obtained with a $14 \mathrm{fs}$ time resolution. (C) 2006 Optical Society of America

OCIS codes: $120.3180,320.2250,320.7100$.
\end{abstract}

Spectral interferometry (SI) has been proposed for retrieving the phase and the amplitude of an unknown electric field. In this technique, two temporally separated coherent pulses interfere spectrally to produce fringes recorded by a spectrometer with sufficient resolution. This technique, coupled with a pumpprobe scheme, can be used for the study of ultrafast dynamics of the laser-matter interaction. ${ }^{1,2}$ This method has been used for many applications, including molecular spectroscopy, ${ }^{3}$ laser-induced dielectric damage, ${ }^{4}$ and laser wakefield (LWF) particle acceleration. ${ }^{5-8}$ However, this method requires multiple shots to construct time-resolved images of the interaction. This aspect is especially critical for the study of the dynamics of electron plasma waves, which is a key phenomenon in LWF particle acceleration, and where terawatt laser pulses are needed. Unfortunately, the pulse-to-pulse stability available from the laser systems currently used to generate such high intensities is not sufficient to obtain repeatable conditions and to reconstruct the complete time history with multiple shots. Recently, singleshot SI with chirped pulses was proposed for solving this problem. ${ }^{9}$ By using a linearly chirped pulse as a probe, rapid temporal changes in phase and amplitude of the probe pulse are encoded at the corresponding temporal frequency. By measuring the fringe shift and intensity change in the spectrum, the perturbed phase and amplitude in the electric field of the probe pulse can be decoded. ${ }^{9}$ However, in this method the time resolution is limited not only by the bandwidth but also by chirp of the probe pulse. ${ }^{10} \mathrm{As}$ was performed in Ref. 11, Fourier analysis is required for retrieving transient phase and amplitude with high temporal resolution. In chirped-pulse SI the perturbed phase and amplitude must be retrieved from the full spectrum, while in standard SI they can be estimated with only a part of the spectrum, since the perturbed phase and amplitude are encoded uniformly in the spectrum. Therefore a higher signal-to-noise ratio can be obtained than with chirped pulse SI. Moreover, to estimate the 2D relative phase distribution, multiple shots are necessary for sweeping the observation slit of the spectrometer to cover the whole objective phase. The $2 \mathrm{D}$ distribution of the relative phase provides more infor- mation than in the 1D case. For example, 2D instability of the electron plasma wave is thought to affect the electron acceleration in the LWF. ${ }^{12}$ Moreover, the $2 \mathrm{D}$ feature is pertinent for observing the LWF in combination with beam shaping techniques ${ }^{7}$ and is of particular interest in the cavitation regime for probing the so-called bubble region. ${ }^{13}$

In this Letter we present a single-shot $2 \mathrm{D}$ plasma measurement technique based on standard SI. A high visibility of interference was successfully obtained with a commercial narrowband filter. The probe pulse was spectrally broadened by a hollow fiber filled with $\mathrm{Ar}$ gas and then recompressed to its near transform limit by a pair of chirped mirrors, setting a constant phase over the frequency domain. The probe pulse is then split in two, and both probe pulses are temporally separated, so as to obtain interference in the frequency domain. The probe beam is then imaged through a narrowband filter onto a CCD, which records the 2D relative phase shift between both probe pulses with a single shot at each probing time.

Here the principle of this method is explained. Let us consider two identical pulses temporally separated by $\tau$. The electric field of each pulse $E_{1}(t), E_{2}(t)$ is expressed by

$$
\begin{aligned}
& E_{1}(t)=\epsilon(t) \exp \left(i \omega_{0} t\right), \\
& E_{2}(t)=\epsilon(t-\tau) \exp \left[i \omega_{0}(t-\tau)\right] \exp (i \Delta \phi) .
\end{aligned}
$$

Here $\epsilon(t)$ is the slowly varying function of the electric field, $\omega_{0}$ the central frequency, and $\Delta \phi$ the relative phase shift between these two pulses. After passing through a narrowband filter, which works as a window function $W(\omega)$, the temporal electric field $E^{\prime}(t)$ is expressed by

$$
E^{\prime}(t)=F^{-1}\left[F\left[E_{1}(t)+E_{2}(t)\right] \cdot W(\omega)\right],
$$

where $F$ and $F^{-1}$ represent Fourier and inverse Fourier transformation operators, respectively. At the detector after the narrowband filter, the detected intensity is given by $I=\int\left|E^{\prime}(t)\right|^{2} \mathrm{~d} t$. If the envelope of both pulses and the window function of the narrowband filter are assumed to be Gaussian, $\epsilon(t)=\epsilon_{0} \exp$ $\left[-(t / \Delta t)^{2}\right], \quad W(\omega)=\exp \left[-\left(\omega-\omega_{0}\right)^{2} / \Delta \omega^{2}\right]$, where $\Delta t$ is pulse duration, the detected intensity becomes 


$$
I \propto 1+\exp \left[-\frac{\tau^{2}}{2\left(\Delta t^{2}+4 / \Delta \omega^{2}\right)}\right] \cos \left(\omega_{0} \tau-\Delta \phi\right) .
$$

This equation shows that both probe pulses can interfere in the time domain through the use of a narrowband filter having a bandwidth $\Delta \omega \ll 1 / \tau$. The exponential factor in the second term in relation (3) approximates to 1 , yielding a visibility of $\sim 100 \%$. In the experiment, $\tau$ is fixed such that $\cos \left(\omega_{0} \tau\right)=-1$, which means that a completely dark image is obtained when no relative phase shift is induced (i.e., when no plasma is generated). Plasma regions inducing a finite $\Delta \phi$ will then appear as bright regions in the $2 \mathrm{D}$ interference image. Therefore the obtained image corresponds to a $2 \mathrm{D}$ distribution of the relative phase shift between both probe pulses. The time evolution of the relative phase shift is obtained by adjusting the delay between the pump pulse and these two probe pulses.

The experiment was performed with an OPCPATi:sapphire hybrid laser system, ${ }^{14}$ which delivers $10 \mathrm{~Hz}, 50 \mathrm{fs}$ pulses at an $800 \mathrm{~nm}$ wavelength. The laser pulse was divided into pump and probe pulses with a beam splitter consisting of a high-reflection mirror with a small hole at the center. The reflected pulse was used as the pump pulse, and the pulse transmitted through the hole was the probe. The experimental setup is shown in Fig. 1. The pump energy was $150 \mathrm{~mJ}$, and it was focused on a supersonic $\mathrm{He}$ gas jet with an $f / 4.6$ off-axis parabolic mirror (OAP). The spot size of the pump pulse at the focal plane was measured to be $7.3 \mu \mathrm{m} \times 8.3 \mu \mathrm{m}$ FWHM in intensity. The orifice diameter of the gas jet nozzle was $2 \mathrm{~mm}$, and the average atomic number density was $8.8 \times 10^{17} \mathrm{~cm}^{-3}$. To obtain sufficient temporal resolution, we injected the probe pulse into an Arfilled hollow fiber where spectral broadening was induced by self-phase modulation. ${ }^{15}$ The bandwidth of the output pulse from the hollow fiber was $100 \mathrm{~nm}$ with $100 \mu \mathrm{J}$ in energy. The output pulse was compressed with a pair of chirped mirrors with a negative group-velocity dispersion of $-35 \mathrm{fs}^{2}$ per reflection. After 32 bounces set to compensate for material dispersion in the setup, the pulse width was mea-

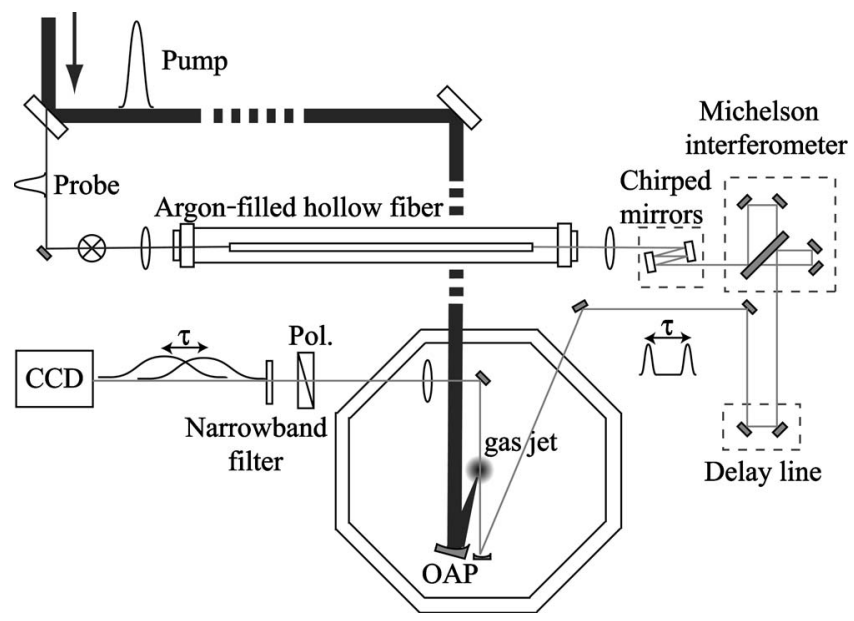

Fig. 1. Experimental setup.
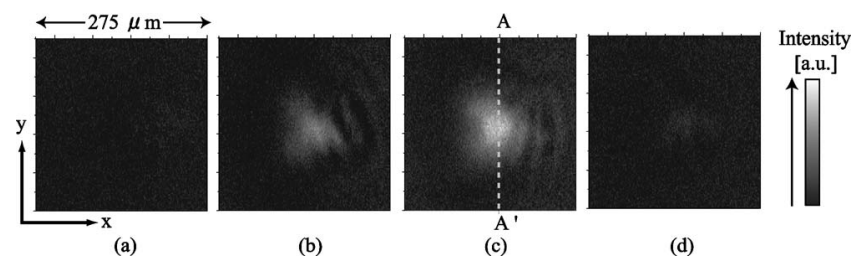

Fig. 2. 2D intensity distribution of the probe beam when the delay between the pump pulse and the second probe pulse is (a) -220 , (b) -13.3 , (c) 0 , or (d) 227 fs. The delay between the two probe pulses was $233 \mathrm{fs}$.

sured by single-shot autocorrelation to be $14 \mathrm{fs}$ FWHM in intensity. This pulse was sent to a Michelson interferometer to generate two collinear probe pulses with adequate temporal separation. The time delay between the probe pulses and the pump pulse was adjusted by a delay line set after the Michelson interferometer, as shown in Fig. 1. The polarization direction of the probe pulses was rotated by $90^{\circ}$ from that of the pump pulse. Probe pulses were focused inside the gas jet with an $f / 410$ lens, and a polarizer was inserted before the CCD to avoid stray light from the pump pulse. Moreover, the injecting direction of the probe beam was tilted by $7^{\circ}$ with respect to the pump pulse. This tilt caused the image to be asymmetric in the horizontal direction. Work is under way to demonstrate that the principle can be applied to the collinear geometry. The focal point of the pump pulse was imaged on the CCD with an $f / 6$ lens. The spatial resolution was $\sim 5 \mu \mathrm{m}$ with a magnification factor of 15. A commercial narrowband filter with a $2.5 \mathrm{~nm}$ spectral width centered at $800 \mathrm{~nm}$ was put in front of the CCD to obtain the spectral interference.

The experimental results are presented in Fig. 2. The image in Fig. 2(a) was obtained when both probe pulses were earlier than the pump pulse. The relative phase shift between the two probe pulses is then spatially uniform. The images of Figs. 2(b) and 2(c) were obtained when the first probe pulse propagated in the gas while the second one propagated in the plasma produced by the pump pulse. These $2 \mathrm{D}$ images correspond to the spatial distributions of the relative phase shift induced on the second probe pulse by the plasma at an interval of $13.3 \mathrm{fs}$. An expansion of the plasma region can be observed during this interval. Afterward, the observed images were similar to that in Fig. 2(c) until both probe pulses propagated in the plasma [Fig. 2(d)]. In comparison with the image of Fig. 2(c), the signal level of the image of Fig. 2(d) drastically decreased. The residual relative phase shift in Fig. 2(d) is thought to be caused by the electron plasma wave excited by the pump pulse, although the temporal reconstruction of the electron plasma wave could not be obtained because of the stability of the laser. The solid curve in Fig. 3 shows the spatial intensity profile along the line $A-A^{\prime}$ in Fig. 2(c). There are three regions that correspond to different charge state populations in the plasma. At $50 \mu \mathrm{m}<|y|<90 \mu \mathrm{m}$, the intensity is lower than the ionization threshold of $\mathrm{He}^{2+}$, and the phase shift is attributed to singly ionized He only. At $15 \mu \mathrm{m}<|y|<50 \mu \mathrm{m}$, the pump intensity is high 


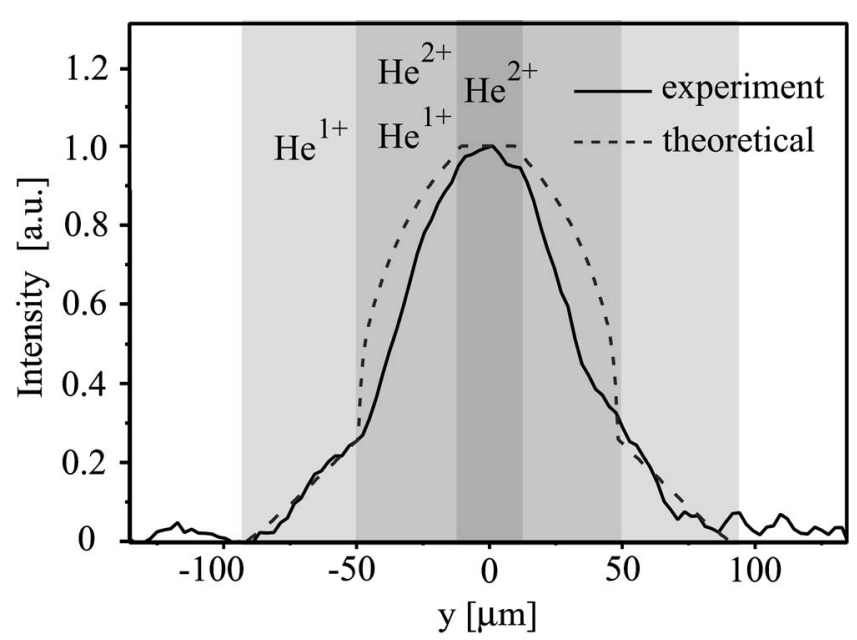

Fig. 3. Intensity distribution along $A-A^{\prime}$, corresponding to the phase difference between the two probe pulses. The inflection point corresponds to the onset of double ionization of He. The dashed curve shows the predicted intensity distribution.

enough to produce $\mathrm{He}^{2+}$ in addition to $\mathrm{He}^{+}$. At $|y|$ $<15 \mu \mathrm{m}$, He was predominantly doubly ionized over the whole region.

Theoretical calculations were performed and compared with the experimental results. The plasma distribution produced by the pump pulse was estimated by using the barrier-suppression ionization model. ${ }^{16}$ $\Delta \phi$ was calculated by integrating the plasma distribution along the propagation direction of the probe pulse, and it was then substituted into Eq. (3). The dashed curve in Fig. 3 shows the calculated profile, which is consistent with the experimental result. The inhomogeneous distribution of ionized $\mathrm{He}$ atoms results in points of discontinuity separating the three regions, also visible in the experimental profile.

In conclusion, we developed a single-shot $2 \mathrm{D}$ spatially resolved SI technique with a narrowband filter. The temporal resolution was improved from previous studies by the combination of spectral broadening with Ar-filled hollow fiber and dispersion compensation with chirped mirrors. This method provides a direct, single-shot, time-resolved measurement of $2 \mathrm{D}$ phase distribution in laser-produced plasmas and is well suited for the observation of ultrafast phenomena, in particular as a diagnostic for LWF acceleration.

The authors thank Akira Suda for providing hollow fibers and useful advice. This research was supported by a Grant-in-Aid for Scientific Research on Priority Areas (grant 14077204) from the Japanese Ministry of Education, Science, Sport and Culture. A. Zoubir was supported by the Japanese Society for the Promotion of Science Postdoctoral Fellowship for Foreign Researchers.

\section{References}

1. E. Tokunaga, A. Terasaki, and T. Kobayashi, Opt. Lett. 17, 1131 (1992).

2. J.-P. Geindre, P. Audebert, A. Rousse, F. Falliès, J.-C. Gauthier, A. Mysyrowicz, A. Dos Santos, G. Hamoniaux, and A. Antonetti, Opt. Lett. 19, 1997 (1994).

3. N. Scherer, R. Carlson, A. Matro, M. Du, A. Ruggiero, V. Romero-Rochin, J. Cina, G. Fleming, and S. Rice, J. Chem. Phys. 95, 1487 (1991).

4. P. Audebert, P. Daguzan, A. Dos Santos, J.-C. Gauthier, J.-P. Geindre, S. Guizard, G. Hamoniaux, K. Krastev, P. Martin, G. Petite, and A. Antonetti, Phys. Rev. Lett. 73, 1990 (1994).

5. J. R. Marquès, J.-P. Geindre, F. Amiranoff, P. Audebert, J. C. Gauthier, A. Antonetti, and G. Grillion, Phys. Rev. Lett. 76, 3566 (1996).

6. C. W. Siders, S. P. Le Blanc, D. Fisher, T. Tajima, and M. C. Downer, Phys. Rev. Lett. 76, 3570 (1996).

7. E. Takahashi, H. Honda, E. Miura, N. Yugami, Y. Nishida, K. Katsura, and K. Kondo, Phys. Rev. E 62, 7247 (2000).

8. H. Kotaki, M. Kando, T. Oketa, S. Masuda, J. K. Koga, S. Kondo, S. Kanazawa, T. Yokoyama, T. Matoba, and K. Nakajima, Phys. Plasmas 9, 1392 (2002).

9. C. Y. Chien, B. La Fontaine, A. Desparois, Z. Jiang, T. W. Johnston, J.-C. Kieffer, H. Pèpin, F. Vidal, and H. P. Mercure, Opt. Lett. 25, 578 (2000).

10. J.-P. Geindre, P. Audebert, S. Rebibo, and J.-C. Gauthier, Opt. Lett. 26, 1612 (2001).

11. K. Y. Kim, I. Alexeev, and H. M. Milchberg, Opt. Express 10, 1563 (2002).

12. E. S. Dodd, R. G. Hemker, C.-K. Huang, S. Wang, C. Ren, W. B. Mori, S. Lee, and T. Katsouleas, Phys. Rev. Lett. 88, 125001 (2002).

13. J. Faure, Y. Glinec, A. Pukhov, S. Kiselev, S. Gordienko, E. Lefebvre, J.-P. Rousseau, F. Burgy, and V. Malka, Nature 431, 541 (2004).

14. K. Kondo, H. Maeda, Y. Hama, S. Morita, A. Zoubir, R. Kodama, K. A. Tanaka, Y. Kitagawa, and Y. Izawa, J. Opt. Soc. Am. B 23, 231 (2006).

15. M. Nisoli, S. De Silvestri, and O. Svelto, Appl. Phys. Lett. 68, 2793 (1996).

16. S. Augst, D. Strickland, D. D. Meyerhofer, S. L. Chin, and J. H. Eberly, Phys. Rev. Lett. 63, 2212 (1989). 